\title{
LA IMPLEMENTACIÓN CURRICULAR DE LA COMPETENCIA GENÉRICA DE TRABAJO EN EQUIPO, DESDE LA EXPERIENCIA DE LOS ESTUDIANTES DE UNA INSTITUCIÓN DE EDUCACIÓN SUPERIOR TÉCNICA
}

\section{CURRICULAR IMPLEMENTATION OF TEAMWORK AS A GENERIC COMPETENCE, FROM THE EXPERIENCE OF THE STUDENTS OF A TECHNICAL HIGHER EDUCATION INSTITUTION}

\author{
Andrea Espejo Beteta ${ }^{1}$ \\ Magíster en Educación con Mención en Currículo \\ Pontificia Universidad Católica del Perú \\ andrea.espejo@pucp.edu.pe
}

Resumen: La investigación se centra en la implementación curricular de la competencia de trabajo en equipo, desde la experiencia de los estudiantes de una institución de educación superior técnica. Se buscó comprender la experiencia de los estudiantes respecto a la enseñanza de la competencia e indagar acerca de su significado para este colectivo. Se trata de un estudio cualitativo de nivel descriptivo y se utilizó un método de corte fenomenológico. El recojo y análisis de información se desarrolló en tres etapas que responden al método: etapa previa de liberación de prejuicios por parte de la investigadora; etapa descriptiva, en base a las entrevistas, se organiza la información y se presenta un análisis por cada sujeto; finalmente, etapa estructural, donde se analiza con una visión holística los resultados obtenidos, contrastándose con el marco conceptual. Se concluye que el currículo vivido por los estudiantes respecto a la competencia de trabajo en equipo está ligado a emociones y percepciones sobre sus habilidades, e importancia y significado que dan a la competencia, siendo para ellos un proceso de múltiples aprendizajes, personales e interpersonales. Desde la enseñanza, manifiestan que se evalúa el producto del trabajo de equipo y no el proceso, siendo dependiente del estilo docente.

Palabras clave: Implementación curricular, competencias, trabajo en equipo, evaluación curricular, estudiantes.

Resumo: A pesquisa tem como foco a implementação curricular da competência do trabalho em equipe, a partir da experiência de alunos de uma instituição de ensino superior técnico. Buscou-se compreender a experiência dos alunos quanto ao ensino de competências e indagar sobre o seu significado para este grupo. Trata-se de um estudo qualitativo de nível descritivo e utilizou-se um método fenomenológico. A coleta e análise das informações foi desenvolvida em três etapas que respondem ao método: etapa anterior de liberação de preconceitos por parte do pesquisador; etapa descritiva, a partir das entrevistas, as informações são organizadas e é apresentada uma análise para cada assunto; por fim, a etapa estrutural, onde os resultados obtidos são analisados com uma

${ }^{1}$ Grupo de Investigación Currículo, Pontificia Universidad Católica del Perú

Fecha recepción: 22 de junio de 2021

Fecha aceptación: 8 de octubre de 2021 
visão holística, contrastando com o quadro conceitual. Conclui-se que o currículo vivido pelos alunos no que se refere à competência do trabalho em equipe está ligado às emoções e percepções sobre suas habilidades, e a importância e o significado que atribuem à competência, sendo para eles um processo de aprendizagem pessoal e interpessoal múltipla. Do ensino, afirmam que é avaliado o produto do trabalho em equipe e não o processo, ficando dependente do estilo de ensino.

Palavras chave: Implementação do currículo, habilidades, trabalho em equipe, avaliação do currículo, alunos.

Abstract: This research focuses on the curricular development of teamwork competence, from the students' experience at a higher technical institution. It was sought to understand the students' experience regarding the teaching of this competence and to inquire about its meaning for the subjects. It is a qualitative study, with a descriptive level and a phenomenological method. The collection and analysis of the information were executed in three stages which correspond with the phenomenological method: a previous stage of the liberation of prejudices on the part of the researcher; a descriptive stage, that consisted of the interviews, and the organization and analysis of information about each subject, and finally, a structural stage, where the results obtained were analyzed with a holistic vision, contrasting it with the conceptual framework and the research problem. It is concluded that the curriculum experienced by the students, regarding the competence of teamwork, is linked to emotions and perceptions about their abilities. Also, the importance and meaning they give to the competence, focusing on the process of an individual and collective learning. Regarding the teaching experience, they stated that the evaluation was focused on the final product, and not so much on the process that involves teamwork competence, always depending on the teaching style.

Keywords: Curricular development, competencies, teamwork, curriculum evaluation, students.

\section{INTRODUCCIÓN}

En las últimas décadas, la competencia de trabajo en equipo ha adquirido cada vez más relevancia tanto desde la mirada formativa como desde los sectores productivos. La Organización para la Cooperación y el Desarrollo Económicos (OCDE) (OCDE, 2005) prioriza esta competencia como una de las claves para llevar una vida exitosa y el buen funcionamiento de la sociedad. Además, la OCDE afirma que las personas dependen de los vínculos que generan con los demás para la supervivencia, así como para la construcción de una identidad social. En ese sentido, las relaciones interpersonales y el aprender a vivir y trabajar con otras personas es fundamental en todo contexto, $y$, por lo tanto, la competencia de trabajo en equipo.

Desde una mirada de enfoque de desarrollo, la competencia de trabajo en equipo se vuelve relevante debido a su priorización dentro de las competencias claves consideradas 
por la OCDE (2005) para una vida exitosa y el buen funcionamiento de nuestra sociedad. La OCDE (2005) considera la interacción en grupos heterogéneos como una categoría de competencia clave, ya que afirma que los seres humanos dependen de sus vínculos con otros para la supervivencia material y psicológica, así como para su identidad social.

Además, en tanto las sociedades se vuelven más fragmentadas y diversas, las relaciones interpersonales se vuelven fundamentales para construir nuevas formas de cooperación. En ese sentido, la competencia de trabajo en equipo se hace necesaria para que las personas aprendan, vivan y trabajen con otros (OCDE, 2005). De acuerdo con esto, Badcock et al. (2010 citado en Dwi, 2017) afirma que: "increasing demand to work in interdisciplinary teams suggests a need for improved interpersonal skills and ability to communicate and work in teams" (p. 41). La priorización entonces de una formación integral de la persona reconoce dicha competencia como fundamental en la educación y, por lo tanto, en el currículo.

La importancia de esta competencia se ha trasladado hacia la educación superior de los distintos países, porque forma parte de la búsqueda de una formación de calidad que integre las demandas de los empleadores y que responda a los sectores productivos, siendo el trabajo en equipo y la comunicación las competencias más requeridas en los profesionales (Cannon-Bowers, 1995, citado en Figl, 2010). Esto porque el trabajo en equipo es una competencia determinante para el éxito profesional y desarrollo personal de los egresados, respecto a su inclusión laboral y social para la OCDE, Comisión Económica para América Latina y el Caribe y Banco de Desarrollo de América Latina (OCDE, CEPAL \& CAF, 2016), ya que implica contar con distintas capacidades como adaptación, flexibilidad y tolerancia, todas relevantes en el contexto actual de cambios permanentes que sufren las diferentes empresas (Pukelis \& Pileičikiené, 2010).

Desde la perspectiva de la oferta formativa en educación superior, para Chan, Fong, Luk y Ho (2017), existe una brecha entre las expectativas de los empleadores respecto a las competencias con las que debe contar un egresado y lo que la oferta educativa actual puede proveer a los estudiantes. Asimismo, hay una brecha distinta entre la oferta educativa y las expectativas de los estudiantes, así como en su comprensión acerca de las competencias genéricas. Además, en dicha oferta formativa, existe la carencia de un marco de enseñanza de competencias genéricas, por lo que los estudiantes no logran concebir claramente las habilidades que los empleadores requieren de ellos. Por estos motivos, el desarrollo de competencias genéricas se ha remediado a través de talleres y otro tipo de actividades fuera del currículo (Chan, Fong, Luk \& Ho, 2017).

Es así como, en la búsqueda de esta correspondencia con las demandas del sector laboral, la educación superior técnica también cobra relevancia en las últimas décadas, tanto a nivel de Latinoamérica como en el Perú, donde el Ministerio de Educación ha incorporado dentro de los requisitos para toda propuesta formativa, la aplicación de un enfoque por competencias, así como el despliegue de competencias genéricas -como el trabajo en equipopara el diseño curricular de cualquier carrera técnica. Este diseño curricular debe estar acompañado de una implementación curricular donde se refleje la enseñanza de dicha 
competencia a través del docente, las actividades realizadas en el aula y, por último, la evaluación de los aprendizajes.

Dicha implementación se refiere también a un currículo realizado en la práctica, también llamado currículo vivido o currículo real (Gimeno, 2010), el cual está conformado por todas las acciones que influyen en los estudiantes tanto dentro como fuera de los espacios formales de aprendizaje. Este conjunto de experiencias y vivencias en distintos contextos son muy importantes para el desarrollo de competencias en los estudiantes, en este caso, la competencia de trabajo en equipo, y ameritan ser estudiadas desde la mirada de ellos mismos y cómo confluyen dichas vivencias en su aprendizaje. Por todo lo expuesto anteriormente es que esta investigación se centra en la implementación curricular de la competencia de trabajo en equipo, y pretende comprender la experiencia de los estudiantes respecto al currículo, a la enseñanza de la competencia y a la percepción que tienen ellos respecto a su propio desarrollo competencial, así como indagar acerca del significado que le asignan dentro de su vida personal y profesional.

La presente investigación se realizó utilizando un método de corte fenomenológico, con el objetivo de obtener un conocimiento más profundo acerca de las experiencias de los estudiantes vinculada a la implementación curricular de la competencia de trabajo en equipo (Ramírez, 2016).

Como principales conclusiones del estudio se encontró que la experiencia de los estudiantes está vinculada a aspectos socioemocionales de la competencia, tanto en sus interacciones con sus compañeros como con sus docentes. Asimismo, el significado que han construido de la competencia está relacionado a un proceso de adquisición de capacidades y actitudes. Además, se encontró que la evaluación de la competencia a nivel institucional ha sido diseñada para evaluar el resultado del equipo en lugar del proceso. Por otro lado, los docentes han jugado un rol importante en el aprendizaje de los estudiantes, desde su propia comprensión de la competencia y de cómo debería ser enseñada, siendo esta distinta para cada docente. Por último, es importante recalcar que esta investigación ha tenido limitaciones vinculadas a la cantidad de entrevistas; no se pudo acceder a más sujetos, lo que hubiese permitido profundizar en los hallazgos de la investigación y alcanzar una mayor comprensión de la esencia del fenómeno.

\section{MARCO DE LA INVESTIGACIÓN}

\section{Implementación curricular de la competencia genérica de trabajo en equipo}

Competencia de trabajo en equipo como competencia genérica

Las competencias genéricas pueden definirse como aquellas capacidades que se encuentran estrechamente vinculadas con la personalidad de los estudiantes y con los tipos de contexto en los que estos se desenvuelven; además, no están vinculadas a situaciones específicas relacionadas a las disciplinas o áreas de conocimiento, lo que le da su carácter de 
genéricas. Para Roegiers (2016), estas competencias se desarrollan sobre todo mientras el sujeto entra en contacto con estímulos durante su formación y cuando interactúa con el entorno y con las demás personas dentro de la institución superior donde curse sus estudios.

De otro lado, las competencias genéricas se relacionan con una serie de dimensiones que incluyen aspectos cognitivos, metodológicos y socioemocionales vinculados al aprendizaje, y que son necesarias para la comprensión y construcción del pensamiento crítico durante la práctica profesional del estudiante (Dejo, 2015; Morita-Alexander, GarcíaRamírez \& Escudero-Nahón, 2016; Suárez, 2017).

Las competencias genéricas, según Pukelis \& Pileičikiené (2010), son esenciales para la competitividad y un buen desempeño en el mercado laboral. En este sentido, estas competencias adquieren una trascendencia disciplinar, así como un carácter o sentido holístico, ya que integran diversos aspectos interrelacionados entre sí (Roegiers, 2016).

Por otro lado, las competencias genéricas contribuyen en la formación integral de los estudiantes, independientemente del área donde se desempeñen, dándoles más posibilidades de hacerlo con éxito durante su vida profesional. Además, al ser transversales a todas las disciplinas, las competencias genéricas pueden ser seleccionadas por la institución como "sellos" que deben concretizarse en todos los planes de estudio, garantizando su desarrollo (Sepúlveda, Quintana, Opazo, Lemarie, \& Sáez, 2013; Rusman \& Dirkx, 2017).

En la Tabla 1 se muestra un comparativo de las principales clasificaciones de competencias genéricas de acuerdo con las fuentes consultadas.

Tabla 1. Principales clasificaciones de las competencias genéricas

\begin{tabular}{|c|c|c|}
\hline Roegiers (2016) & $\begin{array}{c}\text { Villarroel y Bruna (2014), basado en } \\
\text { Tuning }\end{array}$ & $\begin{array}{l}\text { OCDE (2001), citado en } \\
\text { Villaroel\&Bruna }\end{array}$ \\
\hline Organización: liderazgo & $\begin{array}{l}\text { Sistémicas: investigación, } \\
\text { aprendizaje, adaptabilidad, el liderazgo, } \\
\text { la autonomía }\end{array}$ & $\begin{array}{l}\text { Comunicación en lengua } \\
\text { materna y extranjera }\end{array}$ \\
\hline $\begin{array}{l}\text { Socio emocionales: trabajo } \\
\text { en equipo }\end{array}$ & $\begin{array}{l}\text { Interpersonales: capacidad crítica, el } \\
\text { trabajo en equipo, el reconocimiento a } \\
\text { la diversidad y multiculturalidad y el } \\
\text { compromiso ético }\end{array}$ & Conocimientos matemáticos \\
\hline $\begin{array}{l}\text { Psico-emocionales: } \\
\text { confianza en sí mismo }\end{array}$ & $\begin{array}{l}\text { Instrumentales: capacidad de análisis, } \\
\text { síntesis, organización, comunicación, } \\
\text { gestión de información y resolución de } \\
\text { problemas }\end{array}$ & $\begin{array}{l}\text { Competencias relacionadas a } \\
\text { ciencia y tecnología }\end{array}$ \\
\hline \multirow{5}{*}{$\begin{array}{l}\text { Funcionales: uso } \\
\text { herramientas digitales }\end{array}$} & \multirow[t]{5}{*}{-} & Competencias digitales \\
\hline & & Aprender a aprender \\
\hline & & $\begin{array}{l}\text { Competencias } \\
\text { interpersonales }\end{array}$ \\
\hline & & Emprendimiento \\
\hline & & Expresión cultural \\
\hline
\end{tabular}

Fuente: "La implementación curricular de la competencia genérica de trabajo en equipo, desde la experiencia de los estudiantes de una institución de educación superior técnica"; Espejo, 2020, p. 12. 
El MINEDU llama a las competencias genéricas "competencias para la empleabilidad", y también las considera fundamentales para el desempeño en diferentes contextos, tanto personales como profesionales, que además promueven que una persona se inserte y se desarrolle en diferentes empleos (MINEDU, 2018).

\section{Competencia genérica de trabajo en equipo en educación superior}

Existen estudios acerca de equipos de trabajo desde 1970, sin embargo, respecto a la competencia de trabajo en equipo, los estudios son mucho más recientes y pocos han llegado a definirla (Treviño \& Abreu, 2018).

La competencia de trabajo en equipo incluye el conocimiento, principios y conceptos de las tareas y del funcionamiento de un equipo eficaz; el conjunto de habilidades y comportamientos necesarios para realizar las tareas eficazmente, sin olvidar las actitudes apropiadas o pertinentes por parte de cada miembro del equipo que promueven el funcionamiento del equipo eficaz. (Cannon-Bowers, 2000, citado en Treviño \& Abreu, 2018, p. 7)

Además, según Ibarra y Rodríguez (2011), la adquisición de la competencia de trabajo en equipo implica desarrollar la capacidad de integrarse, colaborar y cooperar de manera activa y eficaz con otros individuos para lograr objetivos comunes, integrando todos los aportes del grupo y respetando las opiniones para el bienestar del equipo.

Igualmente, el desarrollo de dicha competencia es un proceso multidimensional, por lo tanto, complejo de analizar y definir; y que abarca otras capacidades del estudiante como organización y establecimiento de objetivos, y a través de la cual se manifiesta la creatividad, empatía, motivación, responsabilidad, compromiso, comunicación, respeto y toma de decisiones como características opuestas al individualismo inherente al ser humano, el cual ha sido promovido tradicionalmente en los sistemas educativos, en búsqueda de competitividad (Jaca et al., 2016; Hernández-Jiménez, 2012; Martínez \& Ion, 2014). Asimismo, para Torrelles et al. (2011), los equipos son distintos y la complejidad del trabajo en equipo implica diferentes grados de dificultad en su desarrollo, los que varían en función al entorno y a otros factores situacionales del equipo.

Según Herrera, Muñoz y Salazar (2017), la competencia de trabajo en equipo promueve la interiorización por parte de los estudiantes de diferentes actitudes que también son muy importantes, como la transparencia, constancia, el compromiso y respeto por los demás. Además, fomenta habilidades dentro de la formación como argumentación, planificación, toma de decisiones y resolución de conflictos. Todo esto puede poner en ventaja a un estudiante que haya tenido dificultades para hablar en público o para argumentar sus ideas.

Por tanto, se puede afirmar que la competencia de trabajo en equipo implica el desarrollo de otras capacidades asociadas como responsabilidad, organización, comunicación, negociación y resolución de conflictos, que, al estar presentes durante la 
interacción y la actividad que se realiza, potencian el funcionamiento de un equipo eficaz. Además, de acuerdo con Herrera et al. (2017), la competencia de trabajo en equipo tiene como características el ser dinámica y multidimensional, que supone principalmente la disposición personal y la colaboración con otros para objetivos comunes. Esto implica la búsqueda de objetivos comunes, el intercambio de información, asignar responsabilidad a los miembros del equipo, resolución de los posibles conflictos que surjan, y la contribución a una mejora y desarrollo colectivo.

De otro lado, el rol de los docentes o tutores que asesoran a los estudiantes durante su trabajo en un equipo es muy importante; por lo que un factor de éxito para el desarrollo efectivo de la competencia es que se disponga de un marco conceptual muy claro que ayude a los docentes a involucrar a los estudiantes en el trabajo en equipo y puedan adquirir la competencia (Jaca et al., 2016). Además, la retroalimentación oportuna a cada estudiante acerca de su comportamiento en el equipo aporta a su desempeño en las próximas oportunidades donde pueda trabajar en un equipo. De acuerdo a ello, existen algunos aspectos que dependen del docente y que afectan el desarrollo de la competencia de trabajo en equipo, como los procesos que ocurren durante la dinámica de los equipos, y la manera en que los docentes evalúan dichos procesos y el desarrollo mismo de la competencia.

De acuerdo con Zabalza (2012 citado en Asún et al.,2019), enseñar una competencia es distinto que emplearla. En ese sentido, la competencia de trabajo en equipo es una de las competencias genéricas que la gran mayoría de docentes buscan desarrollar pero que en la práctica no se toma en cuenta en los procesos de enseñanza y aprendizaje relacionados a las habilidades que se necesitan para el desarrollo de dicha competencia (Asún, Rapún \& Romero, 2019). Las investigaciones sobre la competencia de trabajo en equipo se han enfocado en cómo mejorar el resultado final, sobre todo porque las calificaciones a los estudiantes suelen reflejar resultados individuales más que grupales, por lo que se propone una integración de procesos de autoevaluación y coevaluación para recoger la valoración tanto del docente como de los estudiantes (Morales, 2008; Proulx, 2009, citados en Asún et al., 2019).

Como se ha mencionado, la competencia de trabajo en equipo es un factor fundamental para el desempeño en un entorno laboral, por lo que no es suficiente para los egresados ser competentes técnicamente. Las competencias de trabajo en equipo y comunicación son las más requeridas por los empleadores. La demanda de incluir estas competencias en el currículo ha sido expresada desde hace varios años por los empleadores y por quienes son responsables del diseño curricular en las instituciones educativas (CannonBowers, 1995, citado en Figl, 2010). Se puede remarcar la importancia de la competencia de trabajo en equipo como competencia genérica, tanto desde la perspectiva de los empleadores como en su incorporación en la oferta formativa de las instituciones de educación superior.

Desde la perspectiva de los empleadores, Pukelis \& Pileičikiené (2010) mencionan los cambios que sufre permanentemente el campo laboral donde se desempeñarán los estudiantes, más aún, encontrándonos en una sociedad del conocimiento, donde el desarrollo de la tecnología y de la información es inevitable. En ese contexto, se hace necesaria una flexibilidad, capacidad de adaptación y capacidad para trabajar con otros por parte de los 
futuros profesionales, quienes deberán desenvolverse en diferentes situaciones y circunstancias, con lo cual, las competencias genéricas como el trabajo en equipo son fundamentales en la educación superior. Asimismo, los empleadores afirman que las competencias cognitivas y socioemocionales como el trabajo en equipo son las que determinan el éxito de un profesional, respecto a su inclusión laboral y social (OCDE et al., 2016).

Asún et al. (2019) afirman que esta competencia no sólo es relevante como competencia, sino que desarrolla capacidades que son requeridas por los empleadores, como dirección, liderazgo y relaciones interpersonales. A partir de la competencia de trabajo en equipo, se adquieren herramientas que, más adelante, son utilizadas para desarrollar tareas con otros, donde es necesario un intercambio de ideas, colaboración, negociación, empatía y argumentación, entre otros.

En ese sentido, desde ambas perspectivas, es importante fortalecer el desarrollo de competencias genéricas en la educación superior, ya que los estudiantes buscan insertarse rápidamente en el mundo laboral, dentro del cual las competencias genéricas son claramente requeridas (Bravo, 2016).

\section{Competencias genéricas y su implementación en el currículo}

El proceso en el que se centra la investigación es en la implementación curricular, vinculado al currículo vivido. Esta es definida por Gimeno (2010) como acciones y actividades que son parte de las prácticas y metodologías de enseñanza y que suman al logro de las competencias. Es el desarrollo del diseño (plano escrito) del currículo. Asimismo, para la Oficina Internacional de Educación de la Oficina Internacional de Educación de la Organización de las Naciones Unidas para la Educación, la Ciencia y la Cultura (UNESCO-IBE, 2013), la implementación curricular consta de actividades de enseñanza y aprendizaje que tienen lugar en la escuela, tanto fuera como dentro de las aulas, a través de la interacción entre alumnos y docentes, poniendo en práctica el plan de estudios, llamándose también al currículo vivido como currículo en acción o currículo enseñado.

En ese sentido, la implementación de competencias genera grandes cambios en la labor de los docentes y por lo tanto representa un desafío dentro de su práctica, ya que deben identificar la mejor forma de enseñar y de evaluar competencias genéricas entendiendo la relevancia de una formación integral para los estudiantes y del impacto de las estrategias didácticas que utilicen en el desarrollo de las mismas (Del Valle, 2010; Agudo, HernándezLinares, Rico y Sánchez (2013).

Respecto a los estudiantes, a partir de una investigación de Del Valle \& Bellot, 2009, citados en Del Valle (2010), y un estudio realizado por Asún et al. (2019), se encontró que existen diferencias significativas en su percepción respecto al logro de competencias genéricas, porque además para ellos existe mucha complejidad en la organización y 
dedicación para trabajar en equipo, así como en la diversidad de personalidades y perfiles de sus compañeros.

\section{Educación superior técnica en Latinoamérica y en Perú}

La educación superior técnica ha sido considerada por varias décadas como la oferta más empobrecida o insuficiente del sistema educativo y la opción para quienes no tienen la oportunidad de acceder a estudios universitarios. Sin embargo, actualmente es considerada una de las herramientas fundamentales para el desarrollo económico y social, y para impulsar la movilidad social, siempre y cuando el diseño y desarrollo de la oferta formativa considere las exigencias laborales de la sociedad (Organización de Estados Iberoamericanos para la Educación, la Ciencia y la Cultura [OEI], 2010).

Asimismo, según la OCDE et al. (2016), la educación técnica es fundamental para el desarrollo de una fuerza laboral especializada con diferentes tipos de competencias. Además, en países emergentes como el nuestro, también es una herramienta que debería responder al mercado laboral que está en constante cambio y promover la cohesión social. Es por esta relevancia que, a continuación, se presentará la situación actual de la educación técnica en la región y en el Perú, considerando datos como la matrícula, el egreso, cantidad de instituciones educativas, y algunos problemas que existen en este sector educativo en la última década.

Hasta hace unos años, la gestión de la educación superior técnica en el Perú ha estado enfocada en el aumento de acceso y cobertura, pero de acuerdo con el Instituto de Evaluación de la OEI (2016), la mejora de la calidad en la educación técnica es una prioridad, que se refleja en las políticas educativas de los países latinoamericanos. Con ello, se busca asegurar la inserción de jóvenes a los sectores productivos de cada país, siendo un elemento clave en la formación para el trabajo.

En ese sentido, la educación técnica profesional de calidad se ha convertido en una preocupación tanto en Perú como en los demás países de la región, debido a la creciente necesidad de una oferta educativa que responda a los sectores productivos de dichos países (Ginestié, 2006). Asimismo, es una prioridad generar políticas educativas que apoyen la educación técnica por su competitividad y la empleabilidad para las personas, así como la contribución a la movilidad social (OEI, 2016).

Desde hace pocos años, en el Perú se ha empezado a evidenciar el esfuerzo por la mejora de la calidad de la educación técnica, desde la promulgación de la Ley de Institutos y Escuelas de Educación Superior y de la Carrera Pública de sus Docentes (Congreso de la República del Perú, 2016), así como en el Catálogo de Oferta Formativa y los Lineamientos Académicos Generales (MINEDU, 2018) para el diseño de un currículo por competencias en Educación Superior Tecnológica. Esto suma a la educación técnica porque ha generado un estándar de diseño con el que debe cumplir toda institución para ofrecer programas profesionales técnicos, siendo las competencias genéricas consideradas como "competencias 
para la empleabilidad" y de carácter obligatorio en los diseños curriculares de la Educación Superior Tecnológica (MINEDU, 2016).

\section{METODOLOGÍA}

\section{Problema, objetivos y categorías de la investigación}

Para estudiar el fenómeno de la implementación en el currículo de la competencia de trabajo en equipo, a través de la comprensión de la experiencia de los estudiantes, se planteó como problema de la investigación la siguiente pregunta general: ¿Cómo ha sido la experiencia de los estudiantes respecto a la implementación curricular de la competencia genérica de trabajo en equipo en una institución de educación superior técnica? Dado el nivel descriptivo de esta investigación, este problema central se complementa con las siguientes interrogantes: ¿Cómo ha sido la experiencia de los estudiantes en las distintas unidades didácticas donde se enseña dicha competencia? ¿Cómo han vivido la enseñanza de dicha competencia en las diferentes unidades didácticas transversales donde se encuentra? ¿Cómo ha aportado a su vida académica el trabajo en equipo según como ellos lo vivencian? ¿qué dificultades han experimentado respecto a su relación con otros? ¿Cómo resultó su experiencia en la unidad didáctica de Trabajo en equipo y Liderazgo? ¿Qué significado tiene para ellos esta competencia?

Para responder a las preguntas de investigación, se plantearon los siguientes objetivos:

a. Comprender la experiencia de los estudiantes respecto a la implementación curricular de la competencia de trabajo en equipo.

b. Indagar acerca del significado de la competencia de trabajo en equipo para los estudiantes.

Se tuvo como categorías preliminares, la experiencia de los estudiantes vinculada a la implementación curricular de la competencia, así como el significado de la competencia de trabajo en equipo, de acuerdo a las vivencias que cada uno tuvo durante su formación. Se entiende por experiencia, aquellas situaciones donde los estudiantes interactúan entre ellos y con sus docentes; formando equipos y desarrollando tareas en función a objetivos comunes, tanto en los espacios de clase como fuera de ellos. Asimismo, cuando se indaga por el significado, se recoge el concepto o la idea que han construido los estudiantes acerca de la competencia de trabajo en equipo a partir de sus experiencias. Durante el proceso de análisis de dicha información, estas categorías se convirtieron en macro categorías, surgiendo seis categorías que se detallan en la sección de resultados. 


\section{Método, técnica e instrumento}

Para la presente investigación, se utilizó un método de corte fenomenológico, ya que este centra su interés en el significado que los sujetos atribuyen a los diversos fenómenos que experimentan dentro de una realidad determinada (McMillan \& Schumacher, 2005). A partir del empleo de este método se pudo obtener un conocimiento más profundo de la naturaleza de las experiencias de los estudiantes en relación a la implementación curricular de la competencia de trabajo en equipo, en su proceso formativo, es decir, desde sus experiencias en el aula, analizando el sentido que ellos dan a sus propias vivencias en la institución (Ramírez, 2016).

El método fenomenológico busca mostrar y comprender la esencia de un campo determinado, es decir, la comprensión del mundo visto desde una interpretación de las situaciones cotidianas de una persona (Leal, 2003). Según Fuster (2019), el método fenomenológico analiza la experiencia significativa a nivel consciente. Es importante entender al fenómeno como parte de un todo, y que se debe abordar dentro de toda la experiencia de la que forma parte. El enfoque se centra en cómo las personas comprenden los significados de las experiencias vividas, cómo las han percibido y qué significados las rodean. De esta manera, el método se alinea con la presente investigación, porque precisamente se profundizó en la experiencia de los estudiantes durante su formación vinculada al trabajo en equipo y qué significado tienen dichas experiencias para ellos.

Ayala (2008, citado en Fuster, 2019) aproxima la fenomenología a los procesos educativos, indicando que esta lleva a una reflexión por parte de quienes intervienen en estos procesos dentro de la comunidad educativa; entre ellos los estudiantes. Esto implica que dicha reflexión sea acerca de su experiencia durante el proceso formativo, para que luego establezcan un sentido e importancia a dicha experiencia. En ese sentido, el interés de la investigación se centró en el significado esencial de las experiencias cotidianas de los estudiantes como parte del fenómeno del trabajo en equipo dentro de la institución educativa, y qué importancia y sentido tuvieron dichas experiencias.

Los informantes, en este caso, fueron los estudiantes de una institución de educación superior técnica, que lleva más de 25 años en el medio. Para su selección se consideraron los siguientes criterios:

- Un estudiante de cada uno de los cinco programas de la institución: Administración de empresas, Administración bancaria, Contabilidad, Marketing y Administración de negocios internacionales. Esto se debe al perfil de estudiantes de cada carrera que, por su naturaleza y competencias, son distintos (por ejemplo, un estudiante de Marketing tiene un perfil distinto al estudiante de Contabilidad en cuanto a su desenvolvimiento y habilidades interpersonales).

- Para vincular lo recogido a la implementación curricular de dicha competencia, se seleccionaron estudiantes que hubieran cursado la unidad didáctica de Trabajo en equipo, la cual se dicta en tercer ciclo.

- Estudiantes matriculados que se encontraran cursando los últimos tres ciclos de su formación durante los periodos 2019-1 y 2019-2. 
- Estudiantes que no se encontraran trabajando al momento de la investigación.

En base a los criterios antes señalados, se seleccionaron a cinco estudiantes, logrando que, a través de la técnica de entrevista conversacional aplicada, nos permitieran comprender la esencia de sus vivencias relacionadas al currículo y a la competencia de trabajo en equipo. El tipo de entrevista fue semiestructurada, ya que esta tiene un carácter conversacional, donde la interacción simbólica evita oprimir a las personas entrevistadas, generando un clima coloquial que facilita la comunicación durante la entrevista (Díaz, 2004, citado en Ozonas \& Pérez, 2004). Se utilizó una guía semiestructurada, ya que a partir de ella se indagó acerca de ítems vinculados a las categorías de investigación, pudiendo aparecer categorías emergentes incluso, con el fin de obtener una perspectiva más completa del escenario del entrevistado, a través de preguntas abiertas (Bisquerra, 2012).

Para la elaboración de esta guía de pautas se consideraron aspectos planteados por Merlino (2013) como el marco conceptual, la cercanía que tiene el entrevistado acerca de los temas a indagar, así como la interrelación del tema con otros temas de la vida del sujeto. En este caso, la competencia de trabajo en equipo es una problemática cercana a los estudiantes, no solo porque lo han experimentado en su formación, sino porque lo aplicarán también posteriormente en su vida profesional, y puede ser un factor determinante para su desempeño exitoso.

Asimismo, para dirigir la conversación de manera exitosa, se construyó el instrumento utilizando como base la estructura de Merlino (2013) a través de círculos concéntricos, que inician desde una contextualización con el tema y la investigación, seguida por la movilización o experiencia cotidiana de los estudiantes respecto a los trabajos en equipo que realizan, su vinculación con profesores dentro del tema, así como el significado y la importancia que le atribuyen a la competencia. El instrumento pasó por juicio de expertos, quienes brindaron una retroalimentación a las preguntas de la guía semiestructurada.

También se realizó una entrevista piloto con otro sujeto que cumpliese los criterios de inclusión, con la finalidad de validar la fácil comprensión de las preguntas, su secuencia y la duración aproximada de la entrevista. A partir de dicho piloto, se modificó el orden de las preguntas para una mayor coherencia y fluidez, se eliminaron algunas para reducir su duración, así como también se ajustó la redacción de las preguntas para su mejor comprensión por parte de los estudiantes.

\section{Proceso de investigación}

Para la presente investigación, se han asumido tres fases planteadas tanto por Fermoso (1988) como por Martínez (2004), las cuales se detallarán a continuación. 


\section{Etapa previa}

Esta primera etapa consistió en la liberación de prejuicios desde el investigador, eliminando presupuestos teóricos. Martínez (2004) describe esta etapa como la clarificación de los sesgos que tuviera el investigador respecto al tema. De acuerdo con ello, en esta etapa se aplicó una reducción fenomenológica o epojé, el cual es un ejercicio que, según Husserl (1986, citado en Leal, 2003), implica una desconexión por parte del entrevistador de los aspectos materiales, fácticos y psicofísicos sobre las vivencias investigadas. También denota un estado de duda y suspensión del juicio (Fermoso, 1988). Este término es parte fundamental del método fenomenológico, ya que implica retirarse de la actitud y posición natural que se tiene hacia las cosas para una posición más consciente; suspendiendo la opinión o juicio de valor acerca de los fenómenos a investigar (Aguirre-García \& JaramilloEcheverri, 2012).

La autora de la presente investigación forma parte del área académica de la institución educativa, ejerciendo la jefatura de las carreras de Administración de empresas y Marketing. Esta posición implica la validación del diseño del currículo, de los materiales académicos, además de una revisión y asignación de docentes según su perfil a las diferentes unidades didácticas, para un posterior acompañamiento y supervisión a su desempeño.

\section{Etapa descriptiva}

Esta etapa consistió en una descripción fenomenológica de la experiencia de los sujetos, implicando una visión del fenómeno lo más imparcial, exacta y precisa posible. Martínez (2004) indica que esta etapa es a nivel descriptivo, donde se expone de la manera más fiel posible, la realidad experimentada por los individuos, en este caso por los estudiantes. Previo a ello, se realizaron las entrevistas con los cinco sujetos, donde se firmó un consentimiento informado, indicándoles acerca de la investigación, grabación de voces y uso confidencial de la información.

Para interpretar y analizar la información, en primer lugar, se transcribieron las entrevistas. En segundo lugar, se colocaron códigos dentro de cada transcripción, vinculados a diferentes temas. En tercer lugar, dichos códigos se organizaron en subcategorías y a su vez en categorías emergentes, las cuales se detallarán en el siguiente capítulo. Una vez obtenidas las categorías, se describió la experiencia de cada sujeto a partir de las mismas, respetando en lo posible, la visión de cada uno de forma exacta y precisa.

\section{Etapa estructural}

En esta etapa, se buscó la comprensión de la esencia de la experiencia de los estudiantes, reconociendo sus limitaciones y comprendiendo el fenómeno en su complejidad (Fermoso, 1988; Martínez, 2004). 
Este análisis estructural no sólo respondió a las categorías sino al sujeto en interacción con los diferentes actores que influyeron en su experiencia, partiendo de un reconocimiento de sí mismo, sus emociones y cualidades, encontrando puntos de relación entre los entrevistados para responder a la pregunta y objetivos de investigación. Es decir, se volcó por escrito lo que los sujetos han vivido, captando la naturaleza del fenómeno a partir de las interacciones que estos tuvieron con diferentes actores y elementos curriculares durante toda su formación. Ello sirvió para organizar la presentación de los resultados generales en el apartado respectivo.

\section{RESULTADOS Y ANÁLISIS}

Para el análisis de la información obtenida a partir de las entrevistas, se ha partido del problema de investigación, acerca de cómo ha sido la experiencia de los estudiantes respecto a la implementación curricular de la competencia genérica de trabajo en equipo, en una institución de educación superior técnica. Asimismo, se ha tomado en cuenta preguntas complementarias vinculadas a la experiencia de los estudiantes en las unidades didácticas, tanto la unidad de Trabajo en equipo como otras, la enseñanza de la competencia desde el acompañamiento del docente, el vínculo y la interacción con sus demás compañeros y, por último, el significado sobre la competencia que han construido y la importancia que le asignan a partir de toda su experiencia.

En cuanto a la organización y análisis de los resultados, se consideran las dos macro categorías y al interior se desarrollan diversos temas que se refieren a las categorías de la investigación. A continuación, se presenta una síntesis según las interacciones del sujeto con sus pares, con los docentes, con el currículo para finalmente llegar al significado que asignan los sujetos a la competencia.

La primera macro categoría es acerca de la experiencia vinculada a la implementación curricular de la competencia, cuya definición se mencionó anteriormente. Dentro de esta, se agrupan cuatro categorías. La primera categoría muestra la interacción con otros estudiantes en el desarrollo de la competencia, dentro de la cual se encontraron subcategorías como la actitud de los otros miembros del equipo, y la percepción del desarrollo de la competencia de sus compañeros. La segunda categoría es respecto al trabajo en equipo e incluye subcategorías como factores que influyeron en la motivación del estudiante para trabajar en equipo, así como la dinámica de un equipo, desde su conformación, los roles de los integrantes, el rol que el sujeto asume en cada equipo, influencia del género, etc.

Respecto a la tercera categoría, hace referencia al currículo vivido por el estudiante, y abarca la experiencia en sus unidades didácticas y las actividades extracurriculares dentro de las cuales ha participado y se ha vinculado con sus compañeros. En tanto, la cuarta categoría está vinculada al rol del docente, desde las indicaciones que brinda a los equipos al inicio, el acompañamiento y la motivación a cada equipo, su actitud y la influencia que tiene a partir de su experiencia profesional. Además, cómo el docente toma decisiones respecto a la evaluación de la competencia de trabajo en equipo en cada unidad didáctica. 
La segunda macro categoría se refiere al significado de la competencia de trabajo en equipo asignado por cada estudiante; dentro de la cual se describen dos categorías. Si bien al inicio del estudio el significado se consideró como una sola categoría; a partir de los resultados, se identificó una categoría emergente: la importancia de la competencia. Por lo tanto, el significado se convierte en una macro categoría que subsume el significado entendido, por un lado, como concepto de la competencia, y, por otro lado, como la importancia de la competencia para los estudiantes.

Entonces, la primera categoría señala el concepto que construye sobre la competencia a partir de las capacidades que identifica necesarias para el trabajo en equipo, dentro de las cuales se encuentran las características deseables y no deseables para un adecuado trabajo en equipo, así como la percepción de su propio desarrollo de la competencia.

Por último, la segunda categoría está vinculada a la importancia de la competencia por parte de cada estudiante, tanto como una visión hacia futuro para su vida profesional, como actualmente dentro de su vida personal, incluso familiar, y con sus compañeros en espacios fuera del instituto.

Es por ello que la lógica del análisis a partir del problema, objetivos y categorías se puede traducir en la Figura 1:

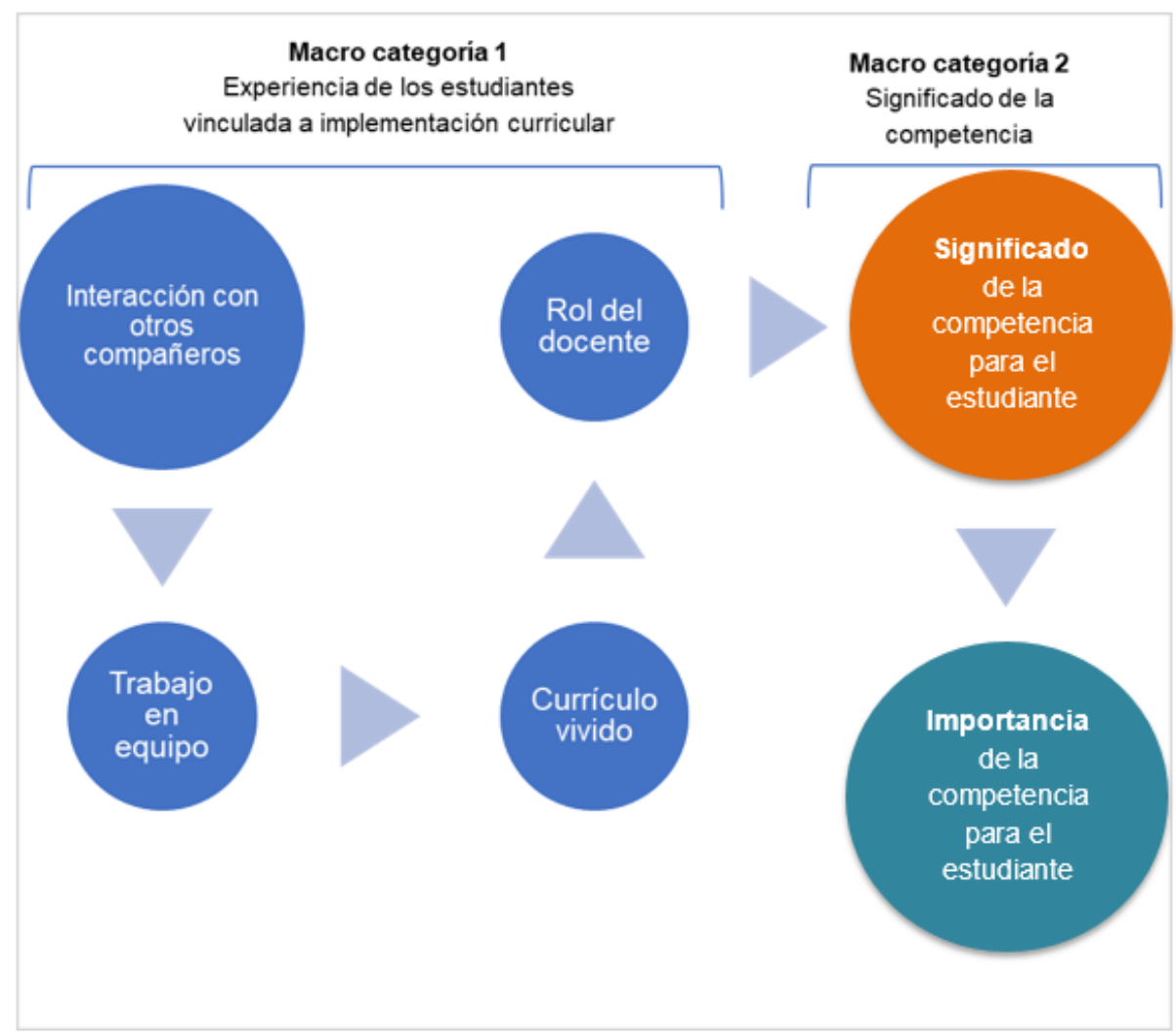

Figura 1. Organización de las categorías para el análisis de la información. Fuente: "La implementación curricular de la competencia genérica de trabajo en equipo, desde la experiencia de los estudiantes de una institución de educación superior técnica"; Espejo, 2020, p. 67. 


\section{Experiencia vinculada a la implementación curricular}

\section{Sobre el sujeto en sí mismo}

Para todos los sujetos, la capacidad de socializar y de establecer relaciones con los demás es clave para trabajar en equipo y para su desarrollo personal, como vencer la timidez, comunicar asertivamente sus ideas, aprender a conocer a nuevas personas; es decir, el tener confianza en ellos mismos y mostrarla ante los demás, ha sido uno de sus principales aprendizajes durante su formación y lo vinculan al aprendizaje de la competencia de trabajo en equipo.

Igualmente, los roles que asumen como parte de un equipo, se transforman en el transcurso de su carrera, si bien todos consideran el liderazgo como el rol ideal, también identifican roles de carácter funcional o de soporte al grupo. Asimismo, existe un reconocimiento de sus propias habilidades y de hacerse cargo de actividades como la investigación, la redacción, o también la iniciativa de dar ideas, motivar a personas que no van al mismo ritmo. Lo principal durante la asignación de su propio rol es el autoconocimiento y la voluntad de aportar con sus habilidades. Esto se relaciona con el estudio realizado por Asún et al. (2019), donde uno de los resultados vinculado a los roles sobre todo tiene que ver con la asunción y ejecución de sus funciones, pero también sobre cómo se han sentido en cada uno de ellos y si les gusta desempeñarlos. En dicho estudio, a diferencia de la presente investigación, los estudiantes no eran conscientes del rol que asumían en el equipo.

Por otro lado, existe una percepción común de que son capaces de trabajar en equipo, es decir, que han adquirido la competencia hacia el final de su proceso formativo; esto se lo atribuyen a la influencia de los docentes, pero sobre todo de las relaciones que han establecido con sus demás compañeros, ya que atribuyen la capacidad de trabajar en equipo a su empatía hacia otros, paciencia, motivación, vínculos de amistad y cercanía. Dicha percepción responde a las capacidades del trabajo en equipo que adquieren los estudiantes, que son características opuestas al individualismo inherente al ser humano (Jaca et al., 2016; Hernández, 2012; Martínez, 2014). Además, porque la competencia requiere de la interiorización de diferentes actitudes como las mencionadas por los estudiantes (Herrera, Muñoz \& Salazar, 2017).

En general, la apreciación sobre las capacidades de cada sujeto sobre sí mismo, vinculadas al desarrollo de la competencia de trabajo en equipo, son principalmente socioemocionales, más que enfocadas en los resultados y en el logro de los objetivos; tal como menciona Asún et al. (2019), para desarrollar la competencia, se debe contar con habilidades para establecer relaciones interpersonales altamente desarrolladas. 


\section{Sobre el sujeto en interacción con sus pares}

"Sentía que trabajaba sola" (sujeto 2). Al comentar acerca de sus demás compañeros, el foco no se encuentra en la calidad del trabajo que cada uno realizaba, sino más bien en actitudes desfavorables que han observado en ellos como falta de comunicación y de colaboración, falta de compromiso, de responsabilidad y poca disposición; y que dichas actitudes generaban conflictos que perjudicaban en gran medida la motivación de los sujetos y el resultado final del equipo. Como indica Treviño, Ninfa, \& Abreu, 2018, la gestión del tiempo, la escucha activa y la colaboración en cada etapa del trabajo es fundamental, y los sujetos no perciben estas características por parte de otros miembros del equipo.

Lencioni (2013 citado por Treviño, Ninfa, \& Abreu, 2018) también menciona la falta de compromiso y la falta de expresión de opiniones, así como la evitación de responsabilidades como reflejo de la falta de compromiso (Natoli, Jackling \& Seelanatha, 2014) lo cual genera desmotivación por parte de quienes son más activos en el equipo, como es el caso de los sujetos entrevistados, y esta desmotivación ante la actitud de los demás. Estos aspectos se han podido identificar a partir de los resultados expuestos por los sujetos.

Asimismo, sólo algunos sujetos mencionan haber aprendido a trabajar en equipo a partir de interactuar con otros compañeros, y quienes lo hacen, se refieren a estudiantes que han actuado como líderes en sus equipos, y que resaltan por sus habilidades para motivar y guiar al resto hacia el resultado. Esto implicaría la necesidad de considerar dentro de la implementación de la competencia, la identificación de estudiantes con capacidad de liderazgo y tenerlo en consideración durante la conformación y dinámica de los equipos en clases.

Por último, el género juega un rol importante dentro de la interacción de los sujetos con los demás. Los hombres distinguen actitudes negativas por parte de las mujeres en sus equipos, y las mujeres distinguen actitudes negativas no solo de los hombres sino también de otras mujeres, en equipos donde todo el grupo es de un solo género o hay una persona de otro género, genera con mayor incidencia discusiones y un clima perjudicial en el equipo.

En general, la dinámica del equipo está mucho más enfocada en cómo los estudiantes manejan las emociones durante los inconvenientes que puedan surgir, y de qué manera enfrentan las actitudes con las que no están de acuerdo por parte de sus compañeros. Estos resultados coinciden con el estudio realizado por Asún et al. (2019) en que para los estudiantes es complejo realizar trabajos en equipo, por la diversidad de personalidades y perfiles de los demás, además de sentir un clima de tensión constante que no favorece su aprendizaje. Tal como afirma Figl (2010), trabajar en equipo muestra a los estudiantes lo beneficios que puede brindar al resultado, sin embargo, todas las experiencias negativas que han vivido, sobre todo vinculadas a la interacción social, determina la predisposición de los estudiantes para trabajar en equipo. 


\section{Sobre el sujeto en interacción con el currículo y la enseñanza}

La experiencia de los estudiantes respecto a los docentes está polarizada, ya que se encuentran expresiones en todos los sujetos acerca de docentes que aportaron al desarrollo de la competencia de trabajo en equipo, mientras que algunos sujetos mencionan solo a un docente que sí fue relevante para dicho desarrollo.

"A los demás docentes no les gusta mucho (...) se hace desorden" (sujeto 5). "Cuando el profesor dice trabajar en equipo, el profesor solo se sienta y ya" (sujeto 1). "Se sentaba en su escritorio y esperaba que termine el tiempo" (sujeto 3). "Otros solo decían que investiguemos por internet y te ponía tu nota" (sujeto 4).

Para los estudiantes, la enseñanza de la competencia implica un docente que los acompañe permanentemente, que brinde retroalimentación; además que pueda entender los conflictos que surjan y oriente al equipo a una solución a través de su experiencia, tal como indica Jaca et al. (2016), el rol del docente y su asesoría al equipo, así como la retroalimentación oportuna es fundamental para el desarrollo de la competencia. Igualmente, las indicaciones del docente previas al trabajo en equipo son importantes para los estudiantes, sin embargo, indican que no siempre estas indicaciones se brindan de manera clara. Esto coincide con Natoli, Jackling \& Seelanatha (2014) quienes mencionan que es necesaria la participación de un asesor que guíe a los estudiantes, sin dejar de considerar la autonomía para resolver los conflictos del equipo.

Para Del Valle (2010) y Rekalde \& Buján (2013), la enseñanza de las competencias genéricas como el trabajo en equipo requiere de una dedicación adicional por parte del docente para preparar las actividades en clase y evaluaciones como parte del despliegue de la competencia, por ello es comprensible que, de acuerdo a la experiencia de los estudiantes, la mayoría de docentes no demostraron una planificación de dichas actividades.

Por otro lado, se encontró que la conformación de los equipos siempre es distinta, y varía según cada unidad didáctica y el docente a cargo, quien pueden determinar que se agrupen de manera voluntaria, establecer grupos de personas que no se conocen, o pedirles que se agrupen de la misma manera como en otras unidades didácticas. Estas formas distintas de agrupación pueden significar oportunidades para los sujetos de interactuar con otros, afirmar sus habilidades socioemocionales o generar mayor conflicto.

Algunos sujetos valoran más las actividades donde deben desenvolverse ante los demás como bailar y actuar, y otros sujetos no las vinculan al desarrollo de la competencia; más bien prefieren agruparse con compañeros que no conocen y realizar trabajos en conjunto. Estas actividades se vinculan con sus experiencias en las diferentes unidades didácticas. Sin embargo, para los sujetos, esta experiencia ha sido marcada por el trabajo y el rol que asume el docente más que por la estructura de las unidades didácticas.

Un ejemplo muy claro de ello es que, a pesar de haber llevado la unidad didáctica de Trabajo en equipo en el mismo periodo, la han vivido de manera particular desde la metodología y actitud de cada docente, aun habiendo desarrollado las mismas dinámicas. 
Para Jaca et al. (2016) el desarrollo de la competencia de trabajo en equipo requiere de un proceso estructurado y transversal en diferentes unidades didácticas. Sin embargo, los estudiantes mencionan que, en otras unidades didácticas, se realizan trabajos principalmente individuales, y, si hubo tareas para realizar en un equipo, la dinámica fue la división de las partes para cada integrante y a continuación la revisión del proyecto consolidado y envío de la tarea. Según Natoli, Jackling \& Seelanatha (2014) esta dinámica genera que cada integrante tenga un entendimiento de algunas partes del trabajo, pero no una visión holística del proyecto realizado. En el caso de la institución donde se realizó el estudio, es una práctica común de los estudiantes a lo largo de su formación, que aparece en la experiencia de estos estudiantes.

Por otro lado, de acuerdo a lo investigado, en entornos virtuales, aún con plataformas colaborativas, los sujetos no han sentido que se logró trabajar en equipo, ya que la comunicación no era oportuna ni adecuada entre sus compañeros, y la falta de puntualidad para conectarse y para presentar avances perjudicaba el logro del resultado. Las plataformas virtuales para trabajar en equipo demandan capacidades que los estudiantes aún están desarrollando como: responsabilidad, autonomía, organización de su tiempo y sus actividades, por lo que quienes pueden desenvolverse en un contexto virtual son la minoría de ellos, y esto genera frustración y rechazo ante estos entornos de aprendizaje.

Respecto a la evaluación de la competencia, todos los sujetos coinciden en que los docentes califican el resultado final, es decir, un producto elaborado y entregado como documento fruto del trabajo en equipo; mientras que para ellos lo más importante fue el proceso por el cual alcanzaron dicho resultado. Asimismo, los docentes evalúan la participación individual durante las clases, lo cual tampoco se relaciona con el desarrollo de la competencia. Dos de los sujetos mencionan que un docente les solicitaba autoevaluaciones y coevaluaciones entre los equipos, previo a la evaluación del docente, pero no tuvieron mayor orientación sobre estas formas de evaluación. Dicho hallazgo responde a lo que indica Del Valle (2010) respecto a la necesidad de que los docentes trabajen las competencias genéricas como el trabajo en equipo de manera consciente con los estudiantes, tomando en cuenta el proceso de desarrollo de dichas competencias para su evaluación. Ello porque las experiencias de los estudiantes durante el proceso han sido aisladas, y al mismo tiempo, no se encuentran fortalecidos los procesos de auto y coevaluación.

\section{Sobre el sujeto en interacción con actividades extracurriculares}

A nivel institucional, se realizan diversas actividades como concursos, talleres, voluntariados, entre otras. Estas tienen el propósito de reforzar distintas competencias que forman parte del perfil de egreso con los estudiantes. Al respecto, ellos mencionan actividades organizadas por cada carrera, como concursos, voluntariados y programas de género. En dichas actividades los sujetos han desarrollado estrategias en equipo, así como interactuar con estudiantes de diferentes carreras que no conocían, con conocimientos distintos, y en el caso de las mujeres, trabajar juntas en proyectos para generar conciencia acerca de la violencia de género. 
Por otra parte, las interacciones que los sujetos observan en otros espacios como la cafetería, áreas de atención al alumno, entre el personal de mantenimiento, para ellos refleja un trabajo en equipo; por ejemplo, la organización de las personas que colaboran en la cafetería para atender a la gran cantidad de personas que se acumulan a la hora de almuerzo. Esto implica que la competencia de trabajo en equipo no solo debe implementarse a través de la planificación de actividades y el rol más preciso de los docentes en el desarrollo de esta competencia; sino también a nivel institucional con las autoridades académicas y administrativas, ya que en estos espacios los estudiantes detectan las dinámicas de los equipos y la actitud de las personas, y ello refuerza el desarrollo de la competencia.

De igual modo, en la implementación de la competencia, se deben tomar en cuenta los diversos espacios de interacción. Por ejemplo, los varones entrevistados comentan acerca de espacios donde juegan en línea con sus compañeros, y en estos videojuegos pueden evidenciar un trabajo en equipo para ganar las partidas, ya que requiere de un alto nivel de coordinación, visión común, ejecución de estrategias y de comunicación permanente. Es importante recalcar que los estudiantes son capaces de tomar conciencia de que esta dinámica promueve su desarrollo de la competencia, por lo que durante el juego pueden detectar ciertas actitudes y comportamientos que necesiten mejorar para trabajar mejor en equipo.

Por último, se ha recogido la importancia de esta competencia para los sujetos en sus relaciones familiares. Es así que todos los sujetos asocian el trabajo en equipo a su vida familiar, sobre todo, en relaciones con personas menores; donde ellos asumen el rol de orientación y guía, como sobrinos y hermanos menores. En estos espacios, los sujetos delegan las tareas y dividen las actividades, y les enseñan a resolver conflictos que puedan surgir durante juegos o discusiones entre ellos.

\section{Significado de la competencia de trabajo en equipo}

\section{Construcción de significado de competencia}

Tal como indica Martínez (2014), Hernández (2012) y Jaca (2016), el desarrollo de la competencia de trabajo en equipo es un proceso multidimensional, lo que la hace compleja de analizar y por lo tanto de definir. Los estudiantes han mencionado diferentes elementos que hacen referencia al significado que ellos han construido de la competencia a partir de todo lo vivido durante su formación y que, efectivamente, abarca distintas dimensiones, desde capacidades hasta actitudes favorables y no favorables.

Como concepto, describen la competencia como un rompecabezas con un solo objetivo; también como una sociedad donde todos dan sus ideas y dichas ideas son respetadas y desarrolladas por el resto. Además, como una colaboración común donde existe un aporte y un aprendizaje por parte de todos los miembros del equipo. Ello resulta interesante en tanto el comprender esta competencia con "rompecabezas" justificaría el que cada quien realiza una parte y el trabajo en equipo es juntar estas partes y alcanzar la meta. 
De otro lado, las otras dos comprensiones como "sociedad" y "colaboración" se acercarían a lo que se espera del ejercicio de la competencia de trabajo en equipo.

Las capacidades que señalan los sujetos coinciden con Martínez (2014), Hernández (2012) y Jaca (2016), como responsabilidad, organización, gestión del tiempo y comunicación. Igualmente, hacen mención a muchas actitudes como las que promueven Herrera et al. (2017), tales como retroalimentación, confianza, respeto, empatía, perseverancia. Sobre todo, hay un énfasis en el liderazgo, entendido como la capacidad de motivar e influir en los demás para que puedan sumar de acuerdo a sus capacidades, así como alentarlos para trabajar bajo presión, y solucionar los conflictos que se puedan presentar para guiar al equipo hacia el objetivo común. De otro lado, las actitudes que los sujetos asumen como desfavorables para el desarrollo de la competencia son la falta de comunicación y el individualismo.

Por último, los estudiantes hablan de otras actitudes que los llevan a generar un vínculo con otros miembros como la solidaridad, amabilidad, el afecto por los otros, hasta estar siempre con una sonrisa ante los demás.

\section{Importancia para su vida profesional}

Todos los sujetos coinciden en la importancia de la competencia para su vida profesional, ya que sirve para el desarrollo de su adaptabilidad, liderazgo, comunicación y colaboración al clima laboral dentro de su centro de trabajo; concordando con CannonBowers (1995, citado por Figl, 2010) quien afirma que el trabajo en equipo y la comunicación son las competencias más requeridas por los empleadores y las que determinarán su éxito profesional. Esto por encontrarnos en un entorno de cambios constantes y requerir de los profesionales dichas competencias para desenvolverse en diferentes situaciones y entre diferentes personas (Pukelis \& Pileičikiené, 2010).

\section{CONCLUSIONES}

Respecto a la comprensión de cómo ha sido la experiencia de los estudiantes vinculada a la implementación curricular de la competencia de trabajo en equipo, se ha encontrado que toda esa experiencia está vinculada a relaciones interpersonales y aspectos socioemocionales de la competencia. Estas relaciones interpersonales han sido con otros compañeros y también con sus docentes; cada una con singularidades y particularidades, que han incidido en su desarrollo personal; no solamente para el aprendizaje de la competencia sino para un reconocimiento de sí mismos como sujetos flexibles ante diferentes contextos y situaciones. En ese sentido, para los estudiantes, el saber ser toma una especial relevancia como componente de la competencia de trabajo en equipo, por encima del saber y del saber hacer (Tobón, 2008). 
Acerca del significado que han construido los estudiantes sobre la competencia de trabajo en equipo, se encontró que el trabajo en equipo significa para todos, un proceso más que la búsqueda de un resultado común que se refleja en un producto; este proceso implica aprendizajes múltiples a nivel personal e interpersonal, no sólo acerca de capacidades sino también un aprendizaje de actitudes y valores para trabajar con otros exitosamente.

Respecto a la enseñanza de la competencia y las actividades realizadas en el aula, existen diferencias en la valoración hacia el docente y hacia el contenido de las unidades didácticas, a pesar de haber llevado la misma unidad didáctica y realizar las mismas dinámicas establecidas previamente en el diseño. Las diferencias se asocian a las indicaciones dadas por el docente, además del acompañamiento y actitud que muestran hacia los estudiantes durante dichas actividades; ya que cada docente mencionado por los sujetos implementa las actividades de manera distinta

De otro lado, a partir de lo comentado por los sujetos, se encuentra que los docentes tienen una distinta comprensión de lo que significa la competencia de trabajo en equipo y de su rol en la formación, ya sea al momento de conformar lo grupos, acompañarlos, dialogar con los estudiantes y organizar las actividades en clase; con lo cual, al ejecutar de distinta manera el currículo diseñado, el nivel de desarrollo de la competencia por los estudiantes se ve afectado.

Respecto a la importancia que asignan los estudiantes a la competencia, se ve reflejada en la manera en que esta ha aportado a su vida, ya que reconocen que, al ser capaces de trabajar en equipo en espacios académicos, son también capaces de trabajar en equipo y de enseñar a otros a hacerlo en espacios como juegos en línea y en espacios familiares donde asumen un rol de guía frente a personas menores que ellos para enseñarles a tomar una actitud colaborativa. 


\section{REFERENCIAS BIBLIOGRÁFICAS}

Agudo, J. E., Hernández-Linares R., Rico, M., \& Sánchez, H. (2013). Competencias transversales: percepción de su desarrollo en el grado en Ingeniería en Diseño Industrial y Desarrollo de Productos. Revista Formación Universitaria, 6(5), 39-50. http://dx.doi.org/10.4067/S0718-50062013000500006

Asún, S., Rapún, M., \& Romero, M. (2019). Percepciones de estudiantes universitarios sobre una evaluación formativa en el trabajo en equipo. Revista Iberoamericana de Evaluación Educativa, 12(1), 175-192. https://doi.org/10.15366/riee2019.12.1.010

Banco de Desarrollo de América Latina. (2016, diciembre 13). USD 150 millones para fortalecer la educación técnica en Panamá con la creación, construcción y puesta en marcha del ITSE. Recuperado de https://www.caf.com/es/actualidad/noticias/2016/12/usd-150-millones-parafortalecer-la-educacion-tecnica-en-panama-con-la-creacion-construccion-y-puestaen-marcha-del-itse/

Bisquerra, R. (Coord.) (2012). Metodología de la investigación educativa. (3a ed.). Madrid: La Muralla.

Bravo, M. G. (2016). Formación de competencias genéricas en estudiantes de enseñanza técnico profesional, desde las percepciones de los docentes. (Tesis de maestría) Recuperado

http://repositorio.uchile.cl/bitstream/handle/2250/146477/Formaci\%C3\%B3n\%20 de $\% 20$ competencias $\% 20$ gen $\%$ C3\%A9ricas $\% 20$ en $\% 20$ estudiantes $\% 20$ de $\% 20$ ense $\%$ C3\%B1anza\%20media\%20t\%C3\%A9cbico\%20profesional\%2c\%20desde\%20las\%2 0percepciones $\% 20 \mathrm{de} \% 20 \mathrm{los} \% 20$ docentes.pdf?sequence $=1 \&$ isAllowed $=\mathrm{y}$

Chan, C., Fong, E., Luk, L., \& Ho, R. (2017). A Review of Literature on Challenges in the Development and Implementation of Generic Competencies in Higher Education Curriculum. International Journal of Educational Development, 57, 1-10. https://doi.org/10.1016/j.ijedudev.2017.08.010

Congreso de la República del Perú. (2016). Ley de Institutos y Escuelas de Educación Superior y de la Carrera Pública de sus Docentes 30512. Diario Oficial El Peruano, 2 de noviembre de 2016. Recuperado de http://busquedas.elperuano.com.pe/normaslegales/ley-deinstitutos-y-escuelas-de-educacion-superior-y-de-la-c-ley-n-30512-1448564-1/

Dejo, N. (2015). Adquisición de competencias en el marco del Aprendizaje Cooperativo: valoración de los estudiantes. REDU, Revista de Docencia Universitaria, 13(1), 339-359. Recuperado de http://red-u.net/redu/files/journals/1/articles/771/public/7713934-1-PB.pdf 
Del Valle, R. (2010). Formación por competencias: certezas, resultados y desafíos. La experiencia de la Universidad Católica de Temuco. II Foro Internacional sobre la Innovación Universitaria. Foro llevado a cabo en México D.F. Recuperado de https://uct.cl/archivos/evaluacion-formacion-competencias-uct.pdf

Dwi, E. (2017) An evaluation of competency-based curricula implemented in the English language education programs in five universities in Indonesia. Global, Urban and Social Studies, $\quad$ RMIT University. $\quad$ Retrieved from http://researchbank.rmit.edu.au/view/rmit:162084

Espejo, A. (2020). La implementación curricular de la competencia genérica de trabajo en equipo, desde la experiencia de los estudiantes de una institución de educación superior técnica. Pontificia Universidad Católica del Perú. http://hdl.handle.net/20.500.12404/16419

Fermoso, P. (1988). El modelo fenomenológico de investigación en pedagogía social. Educar, 1988(14-15), 121-136. https://doi.org/10.5565/rev/educar.541

Figl, K. (2010). A Systematic Review of Developing Team Competencies in Information Systems Education. Journal of Information Systems Education, 21(3), 323-337. Recuperado de http://jise.org/Volume21/n3/JISEv21n3p323.pdf

Fuster, D. E. (2019). Investigación cualitativa: Método fenomenológico hermenéutico. Propósitos y Representaciones. Revista de Psicología Educativa. 7(1), 201-229. http://dx.doi.org/10.20511/pyr2019.v7n1.267

Gimeno, J. (2010). ¿Qué significa el currículum? En: J. Gimeno (Comp.), Saberes e Incertidumbres sobre el Currículum (pp. 21-43). Madrid: Morata.

Ginestié, J. (Coord.). (2006). Systèmes éducatifs et formation des enseignants. Au-delà des apparences, quelles différences ? Une étude internationale sur la formation des enseignants en éducation technologique. Santiago de Chile: Salesianos S.A. Recuperado de

https://www.academia.edu/5422366/Syst\%C3\%A8mes_\%C3\%A9ducatifs_et_format ion_des_enseignants

Ibarra, M. S., \& Rodríguez, G. (2011). Aprendizaje autónomo y trabajo en equipo: reflexiones desde la competencia percibida por los estudiantes universitarios. Revista Electrónica Interuniversitaria de Formación del Profesorado, 14(4), 73-85. Recuperado de http://www.redalyc.org/articulo.oa?id=217022117006

Hernández-Jiménez, D. (2012). Construcción del significado de la evaluación de un programa académico de Ingeniería: Una aproximación fenomenológica. Acta Académica. 2012(51), 63-90. 
Herrera, R. F., Muñoz, F. C., \& Salazar, L. A. (2017). Diagnóstico del trabajo en equipo en estudiantes de Ingeniería en Chile. Formación Universitaria, 10(5), 49-61. https://doi.org/10.4067/S0718-50062017000500006

Jaca, C., Viles, E., \& Zárraga-Rodríguez, M. (2016). Desarrollo de la competencia de trabajo en equipo en un grado universitario. Memoria Investigaciones en Ingeniería, 2016(14), 2334. Recuperado de http://revistas.um.edu.uy/index.php/ingenieria/article/view/333

Leal, N. (2000). El método fenomenológico: principios, momentos y reducciones. Revista Electrónica de Investigación Científica, Humanística y Tecnológica. 1(5), 51-60. Recuperado de http://revistadip.una.edu.ve/volumen1/epistemologia1/lealnestorepistemologia.pdf

Martínez, M. (2004). Ciencia y arte en la metodología cualitativa. México D.F.: Trillas.

Martínez, S., \& Ion, G. (2014). How to Teach and Assess the Teamwork Competency Trough E-Learning? En: I. Roceanu (Ed.), Let's Build the Future through Learning Innovation. Proceedings of the 10th Scientific Conference "eLearning \& Software for Education". Volume 2. (pp. 345-349). Bucharest: Editura Universitatii Nationale de Aparare "Carol I". https://doi.org/10.12753/2066-026X-14-107

McMillan, J. H., \& Schumacher, S. (2005). Investigación educativa. (5a ed.). Madrid: Pearson Educación.

Merlino, A. (2013). La entrevista en profundidad como técnica de producción discursiva. En: A. Merlino (Coord.). Investigación cualitativa en ciencias sociales: temas, problemas y aplicaciones (pp. 111-132). Buenos Aires: Cengage Learning.

Ministerio de Educación de Perú. (2018). Resolución Viceministerial Nº 178-2018-MINEDU. Aprueban el "Catálogo Nacional de la Oferta Formativa de la Educación Superior Tecnológica y Técnico - Productiva. Diario Oficial El Peruano. 9 de noviembre de 2018. Recuperado de https://busquedas.elperuano.pe/download/url/aprueban-el-catalogonacional-de-la-oferta-formativa-de-la-resolucion-vice-ministerial-n-178-2018MINEDU-1710944-1

Ministerio de Educación de Perú. (2016). Guía para la elaboración del plan de estudios. Educación Superior Tecnológica. Recuperado de http://repositorio.MINEDU.gob.pe/handle/123456789/4628

Morita-Alexander, A., García-Ramírez, M. T., \& Escudero-Nahón, A. (2016). Análisis de la percepción de las competencias genéricas en instituciones de educación superior en México. Revista de Educación y Desarrollo, 2016(38), 69-78 Recuperado de http://www.cucs.udg.mx/revistas/edu_desarrollo/anteriores/38/38_Morita.pdf

Natoli, R., Jackling, B., \& Seelanatha, L. (2014). The Impact of Instructor's Group Management Strategies on Students' Attitudes to Group Work and Generic Skill Development. 
Pedagogies: An International Journal, 9(2), 116-132. https://doi.org/10.1080/1554480X.2014.912519

Organización de Estados Iberoamericanos para la Educación, la Ciencia y la Cultura. (2010). Metas Educativas 2021. La educación que queremos para la generación de los bicentenarios. Madrid: Autor.

Organización de Estados Iberoamericanos para la Educación, la Ciencia y la Cultura, Instituto de Evaluación. (2016). Estudio comparado de los sistemas de Educación Técnica de los países de la Alianza del Pacífico. Santiago de Chile: Autor.

Organización de las Naciones Unidas para la Educación, la Ciencia y la Cultura. (2016a). Estrategia para la enseñanza y formación técnica y profesional (EFTP) (2016-2012). Paris: Autor. Recuperado de https://unesdoc.unesco.org/ark:/48223/pf0000245239_spa

Organización de las Naciones Unidas para la Educación, la Ciencia y la Cultura, Oficina Internacional de Educación. (2013). Glossary of Curriculum Terminology. Recuperado de

http://www.ibe.unesco.org/fileadmin/user_upload/Publications/IBE_GlossaryCurric ulumTerminology2013_eng.pdf

Organización para la Cooperación y el Desarrollo Económicos. (2005). Definition and Selection of Key Competencies. Executive Summary. París: OECD Publishing. Recuperado https://www.deseco.ch/bfs/deseco/en/index/02.parsys.43469.downloadList.2296.D ownloadFile.tmp/2005.dskcexecutivesummary.en.pdf

Organización para la Cooperación y el Desarrollo Económicos. (2016). OECD Skills Strategy Diagnostic Report: Peru 2016. París: OECD Publishing. https://doi.org/10.1787/9789264300293-en

Organización para la Cooperación y el Desarrollo Económicos, Comisión Económica para América Latina y el Caribe \& Banco de Desarrollo de América Latina (2016). Perspectivas económicas de América Latina 2017: Juventud, competencias y emprendimiento. París: OECD Publishing. Recuperado de http://dx.doi.org/10.1787/leo-2017-es

Ozonas, L., \& Pérez, A. (2004). La entrevista semiestructurada. Notas sobre una práctica metodológica desde una perspectiva de género. Revista de Estudios de la Mujer, 2004(9), 198-203. Recuperado de http://www.biblioteca.unlpam.edu.ar/pubpdf/aljaba/n09a19ozonas.pdf

Pukelis, K., \& Pileičikiené, N. (2010). Improvement of Generic Skills Development in Study Programmes of Higher Education: The Graduates' Viewpoint. The Quality of Higher Education, 7, 108-131. Recuperado de https://files.eric.ed.gov/fulltext/EJ900261.pdf 
Ramírez, C. A. (2016). Fenomenología hermenéutica y sus implicaciones en enfermería. Index de Enfermería. 25(1-2), 82-85. Recuperado de http://scielo.isciii.es/scielo.php?script=sci_arttext\&pid=S1132$12962016000100019 \& \operatorname{lng}=e s \& \operatorname{lng}=e s$.

Rekalde, I. \& Buján, K. (2013) Las eRúbricas ante la evaluación de competencias transversales en Educación Superior. Revista Complutense de Educación, 25(2) 355-374. Recuperado de https://dialnet.unirioja.es/servlet/articulo?codigo $=4813931$

Roegiers, X. (2016). Marco conceptual para la evaluación de competencias. Ginebra: Organización de las Naciones Unidas para la Educación, la Ciencia y la Cultura, Oficina Internacional de Educación. Recuperado de http://unesdoc.unesco.org/images/0024/002451/245195s.pdf

Rusman, E., \& Dirkx, K. (2017). Developing rubrics to assess complex (generic) skills in the classroom: How to distinguish skills' mastery levels? Practical Assessment, Research and Evaluation. 22.1-9. Retrieved from https://www.researchgate.net/publication/322295240_Developing_rubrics_to_asses s_complex_generic_skills_in_the_classroom_How_to_distinguish_skills'_mastery_levels

Sepúlveda, A., Quintana, M., Opazo, M., Lemarie, R., \& Sáez, D. (2013). Nivel de promoción de competencias genéricas-sello institucionales en educación básica, parvularia y diferencial: percepción de los estudiantes. Revista de Orientación Educacional, 27(52), 75-87. Recuperado de https://dialnet.unirioja.es/servlet/articulo?codigo=5509190

Suárez, X. A. (2017). Percepción de adquisición de competencias genéricas en estudiantes de Psicología que inician y finalizan su formación. Revista Actualidades Investigativas en Educación, 17(3), 1-21. https://doi.org/10.15517/aie.v17i3.30277

Tobón, S. (2008). La formación basada en competencias en la educación superior: el enfoque complejo. Guadalajara: Universidad Autónoma de Guadalajara. Recuperado de http://fcqi.tij.uabc.mx/documentos2010-

2/VideoTutor\%20Modelo\%20Educativo\%20UABC/Ramas/data/downloads/compet encias.pdf

Torrelles, C. et al. (2011). Competencia de trabajo en equipo: Definición y categorización. Profesorado, Revista de Currículum y Formación de Profesorado, 15(3), 329-344. Recuperado de http://www.redalyc.org/articulo.oa?id=56722230020

Treviño, N., \& Abreu, J. L. (2018). Análisis de la problemática del trabajo en equipo en México. Daena: International Journal of Good Conscience. 13(1), 1-13. Recuperado de http://www.spentamexico.org/v13-n1/A1.13(1)1-13.pdf 
Villarroel, V., \& Bruna, D. (2014). Reflexiones en torno a las competencias genéricas en educación superior: Un desafío pendiente. Psicoperspectivas. 13(1), 23-34. https://doi.org/10.5027/psicoperspectivas-Vol13-Issue1-fulltext-335 